\title{
An analysis of risk factors and adverse events in ambulatory surgery
}

This article was published in the following Dove Press journal:

Ambulatory Anesthesia

18 June 2014

Number of times this article has been viewed

\section{Christopher Kent \\ Julia Metzner \\ Laurent Bollag}

Department of Anesthesiology and Pain Medicine, University of Washington Medical Center, Seattle, WA, USA
Correspondence: Julia Metzner Department of Anesthesiology and Pain Medicine, University of Washington Medical Center, 1959 Northeast Pacific Street - Box 356540, Seattle, WA 98195, USA

Tel +l 2065984260

Email metznj@uw.edu
Abstract: Care for patients undergoing ambulatory procedures is a broad and expanding area of anesthetic and surgical practice. There were over 35 million ambulatory surgical procedures performed in the US in 2006. Ambulatory procedures are diverse in both type and setting, as they span the range from biopsies performed under local anesthesia to intra-abdominal laparoscopic procedures, and are performed in offices, freestanding ambulatory surgery centers, and ambulatory units of hospitals. The information on adverse events from these varied settings comes largely from retrospective reviews of sources, such as quality-assurance databases and closed malpractice claims. Very few if any ambulatory procedures are emergent, and in comparison to the inpatient population, ambulatory surgical patients are generally healthier. They are still however subject to most of the same types of adverse events as patients undergoing inpatient surgery, albeit at a lower frequency. The only adverse events that could be considered to be unique to ambulatory surgery are those that arise out of the circumstance of discharging a postoperative patient to an environment lacking skilled nursing care. There is limited information on these types of discharge-related adverse events, but the data that are available are reviewed in an attempt to assist the practitioner in patient selection and discharge decision making. Among ambulatory surgical patients, particularly those undergoing screening or cosmetic procedures, expectations from all parties involved are high, and a definition of adverse events can be expanded to include any occurrence that interrupts the rapid throughput of patients or interferes with early discharge and optimal patient satisfaction. This review covers all types of adverse events, but focuses on the more severe adverse events that are associated with morbidity requiring unplanned admission to hospital and mortality.

Keywords: outpatient surgery, complications

\section{Unplanned admissions after ambulatory surgery}

The cost savings and scheduling advantages of ambulatory surgery are to a large extent lost when patients require unplanned admission to hospital. This makes unplanned admissions a well-studied, theoretically sound starting point for the exploration of adverse events in ambulatory surgery. Unplanned admissions do however result from factors other than adverse events, eg, surgery ending late in the day or lack of a home caregiver, and for that reason any individual instance of hospital transfer while maintaining its status as an adverse economic and administrative event can only be considered a potential marker of an adverse health event.

A recent study from Canada used a case-control method to examine the patient and procedural factors associated with unplanned hospital admissions. ${ }^{1}$ From an ambulatory surgery database of over 20,000 encounters, 200 randomly selected patients who 
were admitted after ambulatory surgery were compared with 200 randomly selected patients who did not require admission. This study did not examine which specific adverse events led to admission, beyond noting that surgical factors accounted for $40 \%$ of admissions, anesthetic factors for $20 \%$, and medical factors for $19 \%$. Social factors and miscellaneous other factors accounted for the remaining admissions. Factors identified as being associated with admission through logistic regression analysis were length of surgery of 1-3 hours (odds ratio [OR] 16.70), length of surgery more than 3 hours (OR 4.26), American Society of Anesthesiologists (ASA) class 3 (OR 4.60), ASA class 4 (OR 6.51), advanced age ( $\geq 80$ years) (OR 5.41), and body mass index (BMI) of 30-35 (OR 2.81). Some of these factors are of questionable clinical relevance for patient selection or other anesthetic decision making, and may reflect some of the limits of this type of analysis, eg, BMI of 30-35 was associated with admission, but higher BMI was not, and cases of intermediate duration had a higher OR for admission than longer cases.

Another Canadian prospective study reported a hospitaladmission rate after ambulatory surgery of $1.4 \%$ from a study population of 15,172 patients. $^{2}$ The indications for admission were classified as surgical in 38\% of cases, anesthetic in $25 \%$, social in $20 \%$, and medical in $17 \%$. More specifically, the three most common types of adverse events that led to the admissions were poorly controlled nausea and vomiting, poorly controlled pain, and procedure-related bleeding requiring treatment or observation. As a combined group of adverse outcomes, major cardiopulmonary events (dysrhythmias, myocardial infarction, heart failure, and angina) accounted for $5 \%$ of admissions and occurred at a frequency of approximately one in every 1,400 procedures.

\section{Patient factors and risk of adverse events in ambulatory surgery}

Three trends in demographics and disease prevalence deserve special attention in an exploration of risk factors for adverse events in ambulatory surgery: the increasing proportion of patients of extreme old age, the increasing prevalence of morbid obesity, and recognition of the increasing occurrence of obstructive sleep apnea (OSA) in the general patient population.

\section{Obesity and obstructive sleep apnea}

Obesity and OSA present a set of independent but frequently overlapping challenges in surgical care. The increasing prevalence of obesity and increasing recognition of OSA have triggered concerns among clinicians regarding the appropriateness of the ambulatory surgical setting for patients with one or both of these conditions.

The largest and most recent study of risk factors in ambulatory surgical procedures utilized the American College of Surgeons' National Surgical Quality Improvement Program (NSQIP) database for the years 2005-2010, analyzing outcomes in over 240,000 patients. $^{3}$ In addition to mortality within 72 hours of surgery, the outcomes included 19 other clinically significant perioperative events and diagnoses, such as pulmonary embolism, cardiac arrest, unplanned intubation, stroke, and surgical site infection. Thirty-eight percent of the patients in the database had a BMI $>30$. Among the 92,008 patients with elevated BMI, $112(0.12 \%)$ experienced an adverse outcome. After multivariate analysis, the patients with BMI $>30$ had an OR of 1.58 for an adverse outcome relative to the patient group with normal BMI, making obesity a statistically significant indicator of adverse outcomes, but not strikingly predictive of their occurrence.

The finding of an increased association between obesity and significant adverse events was not repeated in a retrospective review of outcomes in 2,370 patients undergoing outpatient surgery for laparoscopic placement of adjustable gastric bands for the treatment of obesity. ${ }^{4}$ Thirty-one percent of patients also met the criteria for or were considered to be at high risk for OSA. Although transient oxygen desaturation below $93 \%$ was common immediately postoperatively, there were no other associated adverse respiratory events. Given that $88 \%$ of the members polled for an ASA expert panel felt that upper abdominal laparoscopic surgery could not be performed safely on an outpatient basis in patients with OSA, ${ }^{5}$ it seems remarkable that the rate of unanticipated admission to hospital was lower than in most other reviews of ambulatory surgery, with only one patient requiring hospital transfer due to nausea that was probably related to swelling around the gastric band. This may have been in large part due to adherence to an aggressive postoperative nausea and vomiting prophylaxis regimen and the use of multimodal analgesia.

With regard to specific analyses of OSA, in a case-control study of elective but not exclusively ambulatory surgical procedures, Liao et al reported that the incidence of postoperative complications in OSA patients was statistically significantly greater: $44 \%$ in the OSA group versus $28 \%$ in the non-OSA group. ${ }^{6}$ The most commonly observed between-group difference was in the incidence of oxygen desaturation to less than $90 \%$, which occurred in $17 \%$ of patients with OSA and $8 \%$ without the diagnosis. These results were similar to those of Stierer et al, who studied a cohort of over 2,000 ambulatory 
surgery patients, $4.4 \%$ of whom had a self-reported diagnosis of OSA; another $4.8 \%$ were not formally diagnosed as having OSA, but were found to be at high risk of OSA based on a survey and prediction model. ${ }^{7}$ In the ambulatory setting there was a slight increase in frequency of perioperative events requiring additional anesthetic management in patients with a diagnosis of or higher propensity to OSA. These events were however limited to slightly lower oxygen saturation in the postanesthesia care unit and an increased frequency of difficult intubation, but there was no association with adverse events that required unplanned admission. Even a study like this of a relatively large number of ambulatory surgical patients should be considered underpowered to look at specific risk factors for adverse events, as there were only eleven patients who required unplanned hospital admission. The indications for the majority of the admissions were for concerns not directly related to anesthetic care; specifically, these included the need for continuous bladder irrigation, surgery more extensive than planned, observation of a flap, and postoperative bleeding. Other factors leading to admission could be considered potentially modifiable by anesthetic management, eg, poorly controlled pain, dizziness, and chest pain, but were not obviously related to airway or pulmonary problems.

Bryson et al authored an historical cohort review that included 674 patients with OSA, and reported no difference between the rate of unplanned admission between patients with OSA and those without. ${ }^{8}$ Additionally, the severity of OSA as measured by polysomnography was not shown to correlate with the rate of admission either. Notably in this study, by the protocol of the participating institutions, selection for day surgery was limited to those patients with OSA who were treated with a positive airway-pressure device and were perceived as committed to continuing its use following surgery.

An earlier case-control study by Sabers et al matched patients with polysomnographically confirmed OSA with controls without OSA by type of anesthesia, age, sex, BMI, surgical procedure, and surgical date. ${ }^{9}$ A review of the perioperative medical records indicated that there was no significant difference in the intraoperative management of OSA and control patients, with the exception of a decreased frequency of use of the laryngeal mask airway in the OSA patients. There was no statistically significant difference in the rate of unplanned hospital admissions (24\% versus $19 \%$ ) between OSA and non-OSA patients. In contrast to Bryson et al's study, only $62 \%$ of the OSA patients in this study were receiving positive airway-pressure treatment preoperatively.
The most common reason for hospital admission in Sabers et al's study was "surgical indication", for $48 \%$ of the admissions in the OSA patient group. Notably uncontrolled pain was listed as a reason for admission in $41 \%$ of the OSA patients and in only $21 \%$ of the controls, which on first analysis might suggest a relative reluctance on the behalf of practitioners to administer higher doses of opioids to patients with OSA in an attempt to avoid events related to respiratory depression. Data on weight-based or absolute dosing for perioperative opioid administration was not available for analysis, but the proportions of patients in both groups receiving specific types of opioid analgesics were reported as being similar. Contrary to what might have been expected in patients with OSA, "episodic arterial oxygen desaturation", even after being classified together with bronchospasm in a category of "other respiratory indications", accounted for only four of 46 admissions.

The growing number of older adults will likely increase the proportion of patients with extreme advanced age presenting for ambulatory surgery. This presumption is based on projections of a two- to threefold increase in the elderly population between 2009 and 2030. ${ }^{10}$ Defining the specific risks of ambulatory surgical care in the elderly is a challenging task, and the impact of advanced age independent of other factors on the occurrence of adverse outcomes is not entirely clear. ${ }^{11}$ Many authors have identified advanced age and ASA physical status as limiting factors for outpatient surgery. A large outcome study performed by Chung et $\mathrm{al}^{12}$ reported that $27 \%$ of 17,638 day-surgery patients were 65 years or older and that the risk of intraoperative adverse cardiovascular events, eg, fluctuation in blood pressure and arrhythmias, increased linearly with age and with the prevalence of associated cardiac conditions. Fleisher et al generated an index for predicting death and hospital admission based on the analysis of over 40,000 ambulatory surgeries. ${ }^{13}$ Their research indicated that patients with a combination of any four of seven factors - patient age greater than 85 years, peripheral vascular disease, operating room time greater than 1 hour, malignancy, positive human immunodeficiency virus status, heart disease, and a requirement for general anesthesia - should have surgery in a facility with the capacity for the provision of inpatient care. In contrast, a retrospective review of 1,647 patients older than 70 years undergoing daycase surgery reported a negligible difference in postoperative complication rates compared to the overall population of patients, despite the fact that $12.5 \%$ of patients were classified as ASA $3 .{ }^{14}$ The large NSQIP database study mentioned earlier in this review contained a univariate analysis that 
generated increased ORs for adverse events for patients aged 71-80 (OR 2.3) and >81 (OR 4.1) years. ${ }^{3}$ However, in the multivariate analysis of the data, advanced age no longer remained a statistically significant independent risk factor.

In light of these findings, it appears that neither older age nor the existence of stable coexisting disease independently increases the incidence of perioperative complications in the ambulatory outpatient, but in combination with other factors advanced age appears to become a marker for increased risk.

\section{The pediatric ambulatory patient}

Currently, the vast majority (up to $70 \%$ ) of pediatric surgery in the US is performed on an ambulatory basis. ${ }^{15}$ Complications in healthy children are uncommon, and less than $1 \%$ of children need to be admitted overnight after a planned day-case procedure. ${ }^{16}$ Risk factors associated with serious perioperative adverse events include younger age, coexisting disease, and type of surgery. Respiratory complications are prevalent in preterm infants, children with upper respiratory tract infection (URI), and those diagnosed with OSA. ${ }^{17}$

In preterm infants, apneic spells longer than 15-20 seconds can be observed, and are often associated with desaturation and bradycardia. Such apneic episodes increase in frequency during stressful situations, which would of course include the postsurgical state. This problem was extensively analyzed through studies conducted in former preterm infants following inguinal hernia repair. Cote et al reported a $25 \%$ incidence of apnea strikingly linked to both gestational and postconceptional (PCA) age, with an incidence of less than $5 \%$ with PCA greater than 60 weeks. ${ }^{18}$ Based on a recent literature review, the former otherwise healthy preterm infants with a PCA above 60 weeks can meet standard discharge criteria. ${ }^{19}$ However, in infants with a PCA less than 44-46 weeks, the authors recommended hospital admission and close monitoring for at least 12 hours for apnea, bradycardia, and hypoxia.

The decision to proceed with an elective ambulatory surgical procedure in a child with URI has always been a controversial issue. In the not-too-distant past, the majority of these cases would have been cancelled automatically and surgery postponed for at least 4-6 weeks after resolution of symptoms. ${ }^{20}$ Today, this practice is neither considered practical nor supported by evidence in the literature. Considering the fact that URIs are very frequent in children less than 5 years old, reaching on average six to eight episodes per year with active symptoms lasting for about 1 week, followed by post-URI airway hyperreactivity for up to 6 weeks, it would be very difficult if not impossible to find a URI-free period for elective surgery. ${ }^{21}$ Moreover, unwarranted deferrals may cause substantial financial and emotional consequences for the child and the family, as well as for the facility and surgical team. Although several cohort-based studies have shown a correlation between URI symptoms and perioperative respiratory adverse events, the complications listed were usually mild and easily managed by a competent pediatric team with no long-term sequelae. ${ }^{22}$ Furthermore, the listed adverse events (oxygen desaturation up to 92\%-95\%, laryngospasm, persistent cough, bronchospasm, and airway obstruction) were qualitatively similar to those in pediatric patients who were free of URI symptoms. ${ }^{23-25}$ In a recent large prospective risk-assessment study, URI was associated with an increased risk for perioperative respiratory adverse events only when symptoms were active or when the URI had occurred in the 2 weeks before the procedure. ${ }^{26}$ In concordance with other studies, the authors identified several independent risk factors for adverse respiratory events, which included anesthesia managed with an endotracheal tube (versus use of a laryngeal mask airway or face mask), history of reactive airway disease, history of parental smoking, surgery involving the airway, and the choice of anesthetic regimen (propofol being better than inhalational agents). In conclusion, the decision to postpone or proceed with elective surgery in children with URI should be made on an individual basis by relying on clinical aspects, provider experience, and social context. The occurrence of adverse respiratory events may be reduced by delaying surgery for 2 weeks after resolution of an uncomplicated URI.

Particular vigilance and care is necessary when a child with confirmed or strongly suspected OSA comes for elective ambulatory surgery. A review of adenotonsillectomy for the treatment of OSA found a significantly higher incidence of respiratory complications in patients less than 3 years of age. ${ }^{27}$ Based on this finding, the authors of this review recommended routine overnight admission for these patients. Considering adenotonsillectomy performed as an ambulatory procedure in pediatric patients with OSA, studies have suggested that children age 1-18 years without complicating factors, such as obesity, neuromuscular disease, or craniofacial abnormalities, and with only mild sleep apnea (fewer than 15 obstructive events per hour) may have improvement (fewer obstructive episodes with desaturation) in their symptoms as early as the first night after surgery, and do not need to be monitored intensively. ${ }^{28}$ However, children with moderate/ severe OSA and additional medical conditions like obesity may actually deteriorate on the day of surgery. These children 
should be carefully monitored overnight, particularly when opioids have been administered for pain control. ${ }^{29}$

\section{Lessons learned from closed claims analysis, mandatory reporting systems, and case reports}

The occurrence of major morbidity and mortality is fortunately very rare in all types of surgical care, and even more so in ambulatory surgery. This makes the study of these types of adverse events challenging if not impossible in a prospective manner. There is little standardization in the classification of adverse events and the duration of postoperative surveillance across databases. Closed claims analysis, case reports, quality-assurance databases, and mandatory reporting systems capture these rare events, but are sometimes lacking solid information on the at-risk or denominator population.

A study by Mathis et al utilizing the NSQIP database deserves a closer look for the wealth of information it contains, including denominator data. ${ }^{3}$ Also of note, it was published recently enough to reflect contemporary surgical and anesthesia practice. This review reported an early ( $<72$ hours postoperative) mortality rate of $0.009 \%$ (one in $11,633)$, a major morbidity rate of $0.1 \%$, and an unplanned admission rate of $1.1 \%$ among the nearly 250,000 patients in the database. The patient and procedural factors associated independently with an increased incidence of perioperative morbidity and mortality were, in descending order: chronic obstructive pulmonary disease (OR 2.39), history of cerebrovascular accident or transient ischemic attack (OR 2.15), BMI >30 (OR 2.02), prior percutaneous coronary intervention/cardiac surgery (OR 1.73), prolonged operative time, ie, $>75$ th percentile duration for that procedure type (OR 1.66), hypertension (OR 1.66), and BMI 25-29.9 (OR 1.58). Of the multiple adverse outcomes studied in the review, the five most common were pneumonia, unplanned intubation, wound disruption, bleeding requiring transfusion, and death within 72 hours postoperatively. The procedures associated most frequently with adverse outcomes were laparoscopic cholecystectomy, abdominal wall hernia repair, and inguinal hernia repair.

The largest database for the analysis of closed malpractice claims in anesthesiology is the ASA Closed Claims Project, which in contrast to the NSQIP database does not have denominator data. With the participation of major malpractice insurance companies covering approximately half of the anesthesiologists in the US, the investigators at the Closed Claims Project have built a database of over 5,000 malpractice-claim cases. The factors determining whether or not a patient will file a malpractice claim go beyond the mere occurrence of an adverse event. The majority of patients who experience an adverse event will not file a claim, and occasionally a claim will be filed in the absence of what unbiased experts would judge to be an adverse event. These cases end up being a biased sample of the adverse events occurring in anesthesia care, as they are more likely to be associated with the most severe adverse outcomes. The last analysis of claims focused specifically on adverse events associated with ambulatory anesthesia was published in 2000, making this report somewhat dated, as it usually takes about 5 years for a claim to be closed, meaning that the majority of the events leading to the claims in this analysis occurred around or before $1995 .{ }^{30}$ In this review, Posner reported on both the types of injuries and the "damaging events" that were recorded for the ambulatory surgery claims. Under the standardized data-collection rubric of the Closed Claims Project, a damaging event is the particular aspect of anesthesia management that led to patient injury. For example, the most common damaging events in the ambulatory anesthesia claims involved management of the respiratory system (21\%), which included difficult intubation and inadequate oxygenation or ventilation. The damaging events are then analyzed for their role in relation to specific injuries. The skewed nature of the severity of injuries associated with closed claims is best demonstrated by the fact that the most common injury among the ambulatory surgery malpractice claims was death. The nonfatal injuries reported in descending order of frequency were nerve injury (16\%), eye injury $(10 \%)$, airway injury $(8 \%)$, brain damage $(7 \%)$, pneumothorax (6\%), emotional distress $(5 \%)$, burns (4\%), headache (4\%), and back pain (3\%).

The results of a focused review of a Canadian closed malpractice-claim database affirmed the importance of taking the problem of unescorted ambulatory surgery patients very seriously. Chung and Assmann reported on two incidents where patients who had received procedures with local anesthesia and very minimal amounts of sedation were discharged without escorts and were involved in significantly injurious motor vehicle accidents when driving away from the surgical facilities. ${ }^{31}$ In one of the claims, the anesthesiologist was found to be negligent for administering sedation in the knowledge that the patient did not have an escort, and in the other both the gynecologist and preoperative nurse were found to be negligent for allowing the procedure to be completed and the patient discharged without an escort. These incidents appear to corroborate information indicating that patients have a significant degree of impairment after 
receiving even short-acting general anesthetics and minimal doses of sedating medications.

In the US on a national level, adverse events associated with office-based surgery are not particularly amenable to study, as the regulations regarding accreditation and reporting for these facilities vary greatly from state to state. In 2008 , the authors of a study utilizing data from the Internetbased quality-assurance reporting system of the American Association for Accreditation of Ambulatory Surgical Facilities reported that only 14 states had mandatory accreditation or licensure of ambulatory surgical facilities. ${ }^{32}$ From 2001 to 2006, this reporting system accumulated mortality data on over 1.1 million surgical procedures and recorded 23 deaths occurring within 30 days of an outpatient procedure. Only one of these deaths was related to an intraoperative anesthetic complication, in a case involving propofol, midazolam, and fentanyl sedation supervised by a surgeon without the assistance of an anesthesiologist or nurse anesthetist. Thirteen of the deaths were due to pulmonary emboli, and these were frequently associated with abdominoplasty procedures. The second-most common cause of death in this study was analgesic medication overdose (three deaths). There was one death that was attributed to respiratory arrest in a patient with sleep apnea that occurred on the first postoperative night in spite of a plan to use a pulse oximeter at home. The pulse oximeter was apparently never used by the patient.

Starling et al studied 10 years of data generated by the mandatory adverse event-reporting requirements of Florida and Alabama to evaluate the safety of office-based surgery in the two states..$^{33}$ This type of database identifies deaths and unplanned admissions, but is less useful for generating denominator data for incidence rates. Their review indicated that cosmetic procedures accounted for $57 \%$ of the deaths and $50 \%$ of the hospital transfers reported. Within the category of cosmetic procedures, those performed under general anesthesia accounted for $67 \%$ of the deaths and $74 \%$ of the hospital transfers. Once again, deaths associated with abdominoplasty appeared prominently in this database. Even in the absence of a solid denominator for calculating incidence, the numbers of deaths that were reported to be associated with liposuction with or without abdominoplasty should serve as a red flag that increases the vigilance of clinicians involved with the care of patients undergoing these procedures.

Internet searches outside PubMed using such terms as "complications", "adverse events", "death after discharge", "lawsuits", and "ambulatory or outpatient surgery" produce thousands of hits, only some of which might be considered informative to the practitioner interested in this area.
Individual case and news reports of adverse events associated with ambulatory surgery are likely to focus on the unusual and the tragic, capturing the attention of the clinician and the public, but whether familiarity with these events can be incorporated in any way into any systematic understanding of the risks associated with ambulatory surgery is uncertain. Reports from the lay media on the Internet have the advantage of immediacy and may represent the leading edge of trends or the tip of the iceberg, but they lack peer review, verifiability, and standardized reporting. One of the potentially important themes uncovered in an informal search for this review was the occurrence of opioid-related respiratory arrests and death shortly after discharge or in the postanesthesia care unit. This had been reported with opioid analgesia after tonsillectomy in a child, ${ }^{34}$ tonsillectomy in a teen, ${ }^{35}$ and in an adult with OSA after knee surgery. ${ }^{36}$ Another widely reported postdischarge death in a healthy young woman combined two risk factors: discharge without an escort, and recovery after abdominoplasty with liposuction. ${ }^{37}$ Other notable aspects of this last case were the lack of recording of the volume of tumescent local anesthesia used and postmortem levels of local anesthetic consistent with local anesthetic toxicity.

\section{The unsuitable candidate for ambulatory surgery}

Many of the studies cited in this review included patients in ASA classes 3 and 4 and patients with risk factors that until recently were thought to preclude ambulatory surgery. It is important to keep in mind that in the absence of a very detailed and explicit outline of which factors or specific comorbidities would have excluded patients from eligibility for outpatient surgery in the participating institutions, the analyses of adverse events are blind to the impact that further easing any restrictions on outpatient-surgery eligibility might have on the occurrence of adverse events. The eligibility of patients with ASA physical status 3 and 4 for ambulatory surgery is predicated upon the assumption that comorbidities are stable and optimized. Preoperative assessment by an anesthesiologist, anesthesia perioperative medicine clinic, or clinician familiar with the patient's history to screen these patients and excellent communication with the surgeon or interventionist are crucial to establishing a plan to ensure safety.

The standard practice in many facilities has been that patients with a history of malignant hyperthermia or with identified susceptibility to malignant hyperthermia are often admitted for postoperative observation. Although facilities providing general anesthesia with volatile agents are required 
to maintain treatment capability for malignant hyperthermia crises, the planning and resources required to treat these patients in the event of a crisis should prompt careful consideration about the optimal location for the provision of care to these patients. After provision of a nontriggering anesthetic, patients who are well educated, have a good understanding of their disease process, and have ready access to medical care may be treated as outpatients by some centers.

Although patients who have a history of sleep apnea or who are obese with stable systemic disease appear to be acceptable candidates for ambulatory surgery, there remains some justifiable concern around treating these patients with moderate and severe disease in the outpatient setting. Even the largest studies in this area cannot generate sufficiently predictive information on risks generated by the interaction between more severe elevations in BMI, severe OSA, opioid analgesia, and noncompliance with continuous positive airway pressure. Clinicians must still make judgments on a case-by-case basis.

\section{Summary}

In recent years, clinicians have pushed the boundaries in outpatient surgery, performing increasingly complex procedures in the ambulatory setting, but in spite of this outpatient surgery remains an exceedingly safe area of surgical practice, with unplanned hospital admissions occurring with a frequency of approximately $1 \%$ and major morbidity occurring with a frequency of approximately $0.1 \%$. OSA appears to be associated with the early occurrence of oxygen desaturation and the requirement for airway interventions, but not with an increase in the frequency of unplanned admission nor a statistically significant association with more severe forms of postoperative morbidity. Obesity is associated with increased frequency of postoperative events, but this has not precluded the safe performance of even laparoscopic bariatric procedures on an ambulatory basis. Broadly defined, older age does not appear to be independently associated with adverse outcomes, but comorbidities that occur more frequently in older patients, such as chronic obstructive pulmonary disease, coronary artery disease, peripheral vascular disease, and hypertension, appear to be associated with increased postoperative morbidity. In pediatric ambulatory surgery, the two factors that appear to demand extra vigilance are the risk for apnea in former preterm infants and of airway morbidity in children of all ages undergoing tonsillectomy and adenotonsillectomy, especially for the treatment of OSA. Isolated case reports reaffirm the importance of maintaining a strict policy of requiring that competent adult escorts accompany patients postoperatively. Intra-abdominal and abdominal wall procedures, particularly abdominoplasty, are associated with the highest frequency of postoperative complications, but the incidence of significant morbidity after these procedures remains very low. Given the infrequent occurrence of adverse events in ambulatory surgery, clinicians need to maintain their vigilance, particularly when a number of procedural and patient risk factors align and intersect in any particular episode of patient care.

\section{Disclosure}

The authors report no conflicts of interest in this work.

\section{References}

1. Whippey A, Kostandoff G, Paul J, Ma J, Thabane L, Ma HK. Predictors of unanticipated admission following ambulatory surgery: a retrospective case-control study. Can J Anaesth. 2013;60:675-683.

2. Fortier J, Chung F, Su J. Unanticipated admission after ambulatory surgery - a prospective study. Can J Anaesth. 1998;45:612-619.

3. Mathis MR, Naughton NN, Shanks A, et al. Patient selection for day case-eligible surgery: identifying those at high risk for major complications. Anesthesiology. 2013;119:1310-1321.

4. Kurrek MM, Cobourn C, Wojtasik Z, Kiss A, Dain SL. Morbidity in patients with or at high risk for obstructive sleep apnea after ambulatory laparoscopic gastric banding. Obes Surg. 2011;21:1494-1498.

5. Gross JB, Bachenberg KL, Benumof JL, et al. Practice guidelines for the perioperative management of patients with obstructive sleep apnea: a report by the American Society of Anesthesiologists Task Force on Perioperative Management of patients with obstructive sleep apnea. Anesthesiology. 2006;104:1081-1093.

6. Liao P, Yegneswaran B, Vairavanathan S, Zilberman P, Chung F. Postoperative complications in patients with obstructive sleep apnea: a retrospective matched cohort study. Can J Anesth. 2009;56: 819-828.

7. Stierer TL, Wright C, George A, Thompson RE, Wu CL, Collop N. Risk assessment of obstructive sleep apnea in a population of patients undergoing ambulatory surgery. J Clin Sleep Med. 2010;6:467-472.

8. Bryson GL, Gomez CP, Jee RM, Blackburn J, Taljaard M, Forster AJ. Unplanned admission after day surgery: a historical cohort study in patients with obstructive sleep apnea. Can J Anaesth. 2012;59: $842-851$.

9. Sabers C, Plevak DJ, Schroeder DR, Warner DO. The diagnosis of obstructive sleep apnea as a risk factor for unanticipated admissions in outpatient surgery. Anesth Analg. 2003;96:1328-1335.

10. White PF, White LM, Monk T, et al. Perioperative care for the older outpatient undergoing ambulatory surgery. Anesth Analg. 2012;114: 1190-1215.

11. Bettelli G. High risk patients in day surgery. Minerva Anestesiol. 2009;75:259-268.

12. Chung F, Mezei G, Tong D. Adverse events in ambulatory surgery. A comparison between elderly and younger patients. Can J Anaesth. 1999;46:309-321.

13. Fleisher LA, Pasternak L, Herbert R, Anderson GF. Inpatient hospital admission and death after outpatient surgery in elderly patients. Arch Surg. 2004;139:67-72.

14. Aldwinckle RJ, Montgomery JE. Unplanned admission rates and post discharge complications in patients over the age of 70 following day-case surgery. Anaesthesia. 2004;59:57-59.

15. Rabbitts JA, Groenewald CB, Moriarty JP, Flick R. Epidemiology of ambulatory anesthesia for children in the United States: 2006 and 1996. Anesth Analg. 2010;111:1011-1015. 
16. Patel RI, Hannallah RS. Anesthetic complications following pediatric ambulatory surgery: a 3-yr study. Anesthesiology. 1988;69: 1009-1012.

17. Collins CE, Everett LL. Challenges in pediatric ambulatory anesthesia: kids are different. Anesthesiol Clin. 2010;28:315-328.

18. Cote CJ, Zaslavsky A, Downes JJ, et al. Postoperative apnea in former preterm infants after inguinal herniorrhaphy. A combined analysis. Anesthesiology. 1995;82:808-822.

19. Walther-Larsen S, Rasmussen L. The former preterm infant and risk of post-operative apnoea: recommendations for management. Acta Anaesthesiol Scand. 2006;50:888-893.

20. Tait AR, Malviya S. Anesthesia for the child with an upper respiratory tract infection: still a dilemma? Anesth Analg. 2005;100:59-65.

21. Aquilina AT, Hall WJ, Douglas RG Jr, Utell MJ. Airway reactivity in subjects with viral upper respiratory tract infections: the effects of exercise and cold air. Am Rev Respir Dis. 1980;122:3-10.

22. Becke K. Anesthesia in children with a cold. Curr Opin Anaesthesiol. 2012;25:333-339.

23. Tait AR, Malviya S, Voepel-Lewis T, Munro HM, Seiwert M, Pandit UA. Risk factors for perioperative adverse respiratory events in children with upper respiratory tract infections. Anesthesiology. 2001;95:299-306.

24. Tait AR, Knight PR. Intraoperative respiratory complications in patients with upper respiratory tract infections. Can J Anaesthesia. 1987;34: 300-303.

25. Mamie C, Habre W, Delhumeau C, Argiroffo CB, Morabia A. Incidence and risk factors of perioperative respiratory adverse events in children undergoing elective surgery. Paediatr Anaesth. 2004;14:218-224.

26. von Ungern-Sternberg BS, Boda K, Chambers NA, et al. Risk assessment for respiratory complications in paediatric anaesthesia: a prospective cohort study. Lancet. 2010;376:773-783.

27. Statham MM, Elluru RG, Buncher R, Kalra M. Adenotonsillectomy for obstructive sleep apnea syndrome in young children: prevalence of pulmonary complications. Arch Otolaryngol Head Neck Surg. 2006;132:476-480.
28. Nixon GM, Kermack AS, McGregor CD, et al. Sleep and breathing on the first night after adenotonsillectomy for obstructive sleep apnea. Pediatr Pulmonol. 2005;39:332-338.

29. Leong AC, Davis JP. Morbidity after adenotonsillectomy for paediatric obstructive sleep apnoea syndrome: waking up to a pragmatic approach. J Laryngol Otol. 2007;121:809-817.

30. Posner KL. Liability profile of ambulatory anesthesia. ASA Newsl. 2000;64:10-12.

31. Chung F, Assmann N. Car accidents after ambulatory surgery in patients without an escort. Anesth Analg. 2008;106:817-820.

32. Keyes GR, Singer R, Iverson RE, et al. Mortality in outpatient surgery. Plast Reconstr Surg. 2008;122:245-250.

33. Starling J 3rd, Thosani MK, Coldiron BM. Determining the safety of office-based surgery: what 10 years of Florida data and 6 years of Alabama data reveal. Dermatol Surg. 2012;38:171-177.

34. McGraw M. Did early discharge cause child's death after tonsillectomy? 2012. Available from: http://www.outpatientsurgery.net/surgicalservices/tonsillectomy/did-early-discharge-cause-child-s-death-aftertonsillectomy--05-04-12. Accessed March 20, 2014.

35. Elliott-Engel A. Montco claim over post-surgery death settles for $\$ 6$ mil. 2013. Available from: http://www.rossfellercasey.com/articles/ LI-01-09-13.pdf. Accessed March 20, 2014.

36. PR Newswire. Childers, Schlueter, \& Smith: Patient dies after routine outpatient knee surgery. 2011. Available from: http://www.prnewswire.com/news-releases/childers-schlueter--smith-patient-dies-afterroutine-outpatient-knee-surgery-121169559.html. Accessed March $20,2014$.

37. Graham Lundberg Peschel. Medical negligence wrongful death claim against Sono Bello and Dr Marco Sobrino settled for \$1.9 million. Available from: http:/www.glpattorneys.com/1876636-79-settlementmedical-negligence-wrongful-death-claim-sono-bello-dr-marcosobrino. Accessed March 20, 2014.
Ambulatory Anesthesia

\section{Publish your work in this journal}

Ambulatory Anesthesia is an international, peer reviewed, open access journal publishing articles that address all aspects of ambulatory anesthesia practice, in particular: anesthetic techniques, sedation and safety practices, pharmacokinetics, preoperative evaluation, analgesia interventions, regulatory and compliance issues, postoperative recovery,

\section{Dovepress}

patient satisfaction, administrative topics, and cost analysis themes. The manuscript management system is completely online and includes a very quick and fair peer review system, which is all easy to use. Visit http://www.dovepress.com/testimonials.php to read real quotes from published authors. 